\title{
Frequency-Dependent Piezoresistive Effect in Top-down Fabricated Gold Nanoresistors
}

\author{
Chaoyang Ti, Atakan B. Ari, M. Çağatay Karakan, Cenk Yanik, Ismet I. Kaya, M. Selim Hanay, \\ Oleksiy Svitelskiy, Miguel González, Huseyin Seren, and Kamil L. Ekinci*
}

Cite This: Nano Lett. 2021, 21, 6533-6539

Read Online

ACCESS | Lill Metrics \& More | 国 Article Recommendations ｜ st Supporting Information
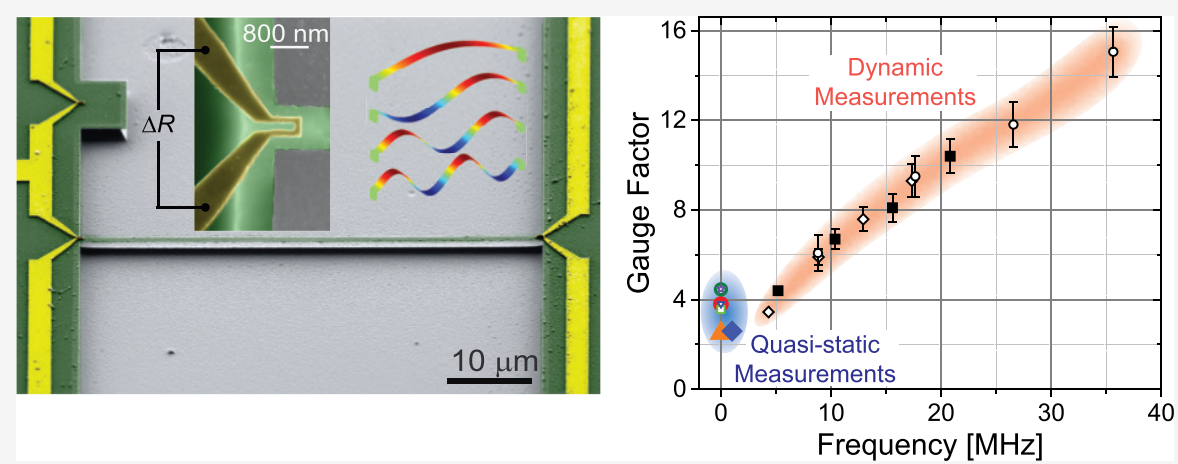

ABSTRACT: Piezoresistive strain gauges allow for electronic readout of mechanical deformations with high fidelity. As piezoresistive strain gauges are aggressively being scaled down for applications in nanotechnology, it has become critical to investigate their physical attributes at different limits. Here, we describe an experimental approach for studying the piezoresistive gauge factor of a gold thin-film nanoresistor as a function of frequency. The nanoresistor is fabricated lithographically near the anchor of a nanomechanical doubly clamped beam resonator. As the resonator is driven to resonance in one of its normal modes, the nanoresistor is exposed to frequency-dependent strains of $\varepsilon \lesssim 10^{-5}$ in the $4-36 \mathrm{MHz}$ range. We calibrate the strain using optical interferometry and measure the resistance changes using a radio frequency mix-down technique. The piezoresistive gauge factor $\gamma$ of our lithographic gold nanoresistors is $\gamma \approx 3.6$ at $4 \mathrm{MHz}$, in agreement with comparable macroscopic thin metal film resistors in previous works. However, our $\gamma$ values increase monotonically with frequency and reach $\gamma \approx 15$ at $36 \mathrm{MHz}$. We discuss possible physics that may give rise to this unexpected frequency dependence.

KEYWORDS: Piezoresistive effect, piezoresistive gauge factor, gold nanowire, gold nanoresistor, NEMS

$\mathrm{T}^{\mathrm{l}}$ he electrical resistance of a bar of metal or semiconductor is typically a function of the mechanical strain on the bar, referred to as piezoresistivity or the piezoresistive effect. ${ }^{1}$ By exploiting this change in resistance with strain, a number of commonly used sensors have been developed for different technological applications and metrology. The fact that piezoresistive strain gauges are scalable in size has allowed for their integration into micro- and nanoelectro-mechanical systems $\left(\mathrm{MEMS}^{2-4}\right.$ and $\mathrm{NEMS}^{5-8}$ ), paving the way for promising technologies. The piezoresistive effect is quantified by the gauge factor, $\gamma=\frac{1}{\varepsilon} \frac{\Delta R}{R}$, which typically relates the "longitudinal strain" $\varepsilon$ to the fractional change in resistance, $\frac{\Delta R}{R}$ 1,6,7,9 For a simple resistor geometry such as a bar, one can write the resistance as $R=\frac{\rho L}{A}$ in terms of the resistivity $\rho$, length $L$, and cross-sectional area $A$ of the resistor. This leads to the well-known expression for the gauge factor, $\gamma=(1+2 \nu)+\frac{1}{\varepsilon} \frac{\Delta \rho}{\rho}$, where $\nu$ is the Poisson's ratio. ${ }^{1}$ Thus, two distinct mechanisms determine the gauge factor. The first term, $(1+2 \nu)$, typically less than 2 , represents a purely geometric effect, ${ }^{10}$ that is, an increase in length and a decrease in the cross-sectional area of the resistor. The second term, $\frac{1}{\varepsilon} \frac{\Delta \rho}{\rho}$, captures the changes in the intrinsic conduction of the material $^{11,12}$ arising from the applied strain.

In this manuscript, our focus is on the piezoresistivity of technologically important thin metal-film resistors. The piezoresistive properties of metal films have been investigated extensively as a function of sheet resistance (thickness), ${ }^{10}$ strain, ${ }^{13,14}$ and structure (i.e., grain size and separation). ${ }^{15}$ In

Received: $\quad$ May 2, 2021

Revised: July 22, 2021

Published: July 28, 2021 
thicker films with low sheet resistances $R_{s} \lesssim 10^{3} \Omega / \square$, the geometric effect dominates, resulting in $\gamma \lesssim 5$. The deviation of $\gamma$ from the purely geometric limit of $\gamma \approx 2$ has been attributed to the increase in the vibrational amplitude of the crystal atoms due to the applied strain; this results in the Gruneisen constant $G$ to enter the expression for $\gamma^{10,15,16}$ as $\gamma=(1+2 \nu)+[1+$ $2 G(1-2 \nu)]$. In ultrathin films with large sheet resistances, $\gamma$ can easily exceed $10^{3},{ }^{10,11}$ suggesting that electron tunneling between grains and through cracks in the film become relevant. Most of the aforementioned measurements of $\gamma$ have been performed using static or relatively low-frequency $(\lesssim 1 \mathrm{MHz})$ strains, ${ }^{17}$ even though strain gauges have been used at frequencies higher than $100 \mathrm{MHz}{ }^{6}$

Looking at all the previous body of work on piezoresistivity of metal films, studies on two important limits remain missing. The first is the piezoresistivity of a metal resistor with nanoscale cross-sectional dimensions. The few studies on nanowires are based on semiconducting nanowires. ${ }^{18-20}$ Second, the frequency dependence of the piezoresistive effect, at the nanoscale or otherwise, has not yet been addressed methodically, possibly due to measurement challenges. Here, we address these questions by measuring the gauge factor of a nanoscale strain gauge as a function of frequency at room temperature. We show that $\gamma$ of our nanoscale strain gauge at 4 $\mathrm{MHz}$ agrees with previous reports on macroscopic gold films at low frequency, suggesting that conduction in our nanoresistor is similar to that in macroscopic films. Our $\gamma$ values, however, increase monotonically with frequency, reaching $\gamma \approx 15$ at 36 $\mathrm{MHz}$.

We perform our study of piezoresistivity of nanoresistors using NEMS resonators such as the one shown in the scanning electron microscope (SEM) image in Figure 1a. This is a tension-dominated silicon nitride doubly clamped beam with linear dimensions of $l \times w \times t \approx 50 \mu \mathrm{m} \times 900 \mathrm{~nm} \times 100 \mathrm{~nm}$. On the two anchor regions of the doubly clamped beam, gold electrodes are patterned using electron beam lithography, thermal film deposition, and lift off. The strain gauge is shown in Figure 1b: this is a $135 \mathrm{~nm}$-thick lithographic u-shaped gold nanowire and is fabricated over the anchor region of the suspended silicon nitride beam (the brighter region in the SEM image in Figure $1 \mathrm{~b}$ ). The strain gauge is "wired" into a bridge circuit along with a nominally identical nanoresistor, as shown in Figure 1c. The circuit diagram in Figure 1c represents the entire bridge circuit embedding the strain gauge and the balancing resistor. Here, $R_{u}$ corresponds to the resistances of the strain gauge and the balancing resistor; $R_{x}$ $R_{y}$, and $R_{z}$ are the lithographic wires connecting the nanoresistors to three $\mathrm{mm}$-scale wirebonding pads; the contact resistance $R_{c}$ corresponds to the wirebonds. ${ }^{21}$ The resistance values for the circuit elements in Figure 1c are calculated from the experimentally measured resistivity $\rho$ of the gold film. To this end, we first make a four-wire measurement of the gold resistor represented by $R_{y}+R_{u}+R_{x}+R_{z}$ and find this resistance to be $14.51 \pm 0.14 \Omega$. We then compute the same resistance from geometry (i.e., SEM images) in terms of an unknown $\rho$ using two methods: (i) we integrate the infinitesimal resistance $d R=\rho \frac{d l}{h W(l)}$ along the electron path using the position-dependent width $W(l)$; (ii) we "count" the number of squares $N$ and determine the total resistance as $N \frac{\rho}{h}$. We find $\rho \approx 2.82 \times 10^{-8} \Omega \cdot \mathrm{m}$. With $\rho$ determined, we calculate the resistance of each individual resistor from its geometry, as reported in Table 1 . There is typically a small

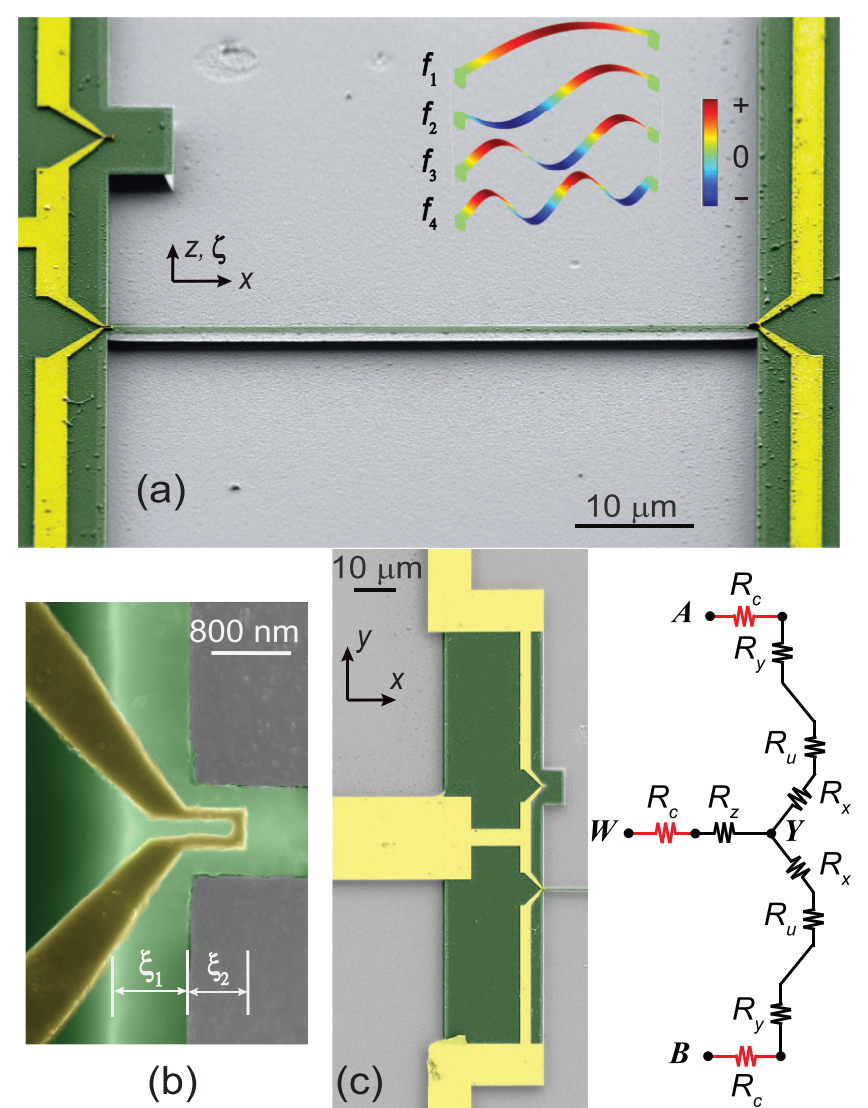

Figure 1. (a) SEM image of a silicon nitride doubly clamped beam with linear dimensions of $l \times w \times t \approx 50 \mu \mathrm{m} \times 900 \mathrm{~nm} \times 100 \mathrm{~nm}$. The gold nanoresistors fabricated on the anchors act as a strain gauge (left) and an electrothermal actuator (right). The eigen-modes of the structure are shown in the finite element simulations in the inset with the color bar corresponding to the out-of-plane displacement. (b) Close-up of the strain gauge. This u-shaped thin film nanoresistor has a thickness of $h=135 \mathrm{~nm}$ and a width of $120 \mathrm{~nm}$. The undercut region $\left(\xi_{1} \approx 800 \mathrm{~nm}\right)$ and the region on the bridge $\left(\xi_{2} \approx 600 \mathrm{~nm}\right)$ are both deformed when the beam vibrates. (c) SEM image of the electrode (left) and the circuit model (right). The strain gauge is balanced with a nominally identical nanoresistor. The resistance of the strain gauge and the balancing resistor is $R_{u} ; R_{x}, R_{y}$, and $R_{z}$ are the resistances of the lithographic wires in different regions of the electrode; $R_{c}$ is the (average) electrical contact resistance from wirebonds.

Table 1. Resistance Values for Each Lithographic Resistor in Device Calculated from Resistivity $\rho$ and Geometry ${ }^{a}$

$\begin{array}{ccccc}R_{x} & R_{y} & R_{u} & R_{z} & R_{c} \\ 2.16 \Omega & 7.37 \Omega & 3.54 \Omega & 1.43 \Omega & 1.18 \Omega \\ { }^{T} & \end{array}$

${ }^{a}$ Typical error in these values is $5 \%$.

mismatch between the two arms of the bridge of about 2-5\%, which contributes to the errors. Subsequent two-wire measurements provide the contact resistances of the wirebonds to be $R_{c}$ $=1.18 \pm 0.07 \Omega$. On the second anchor of the NEMS beam (right anchor in Figure 1a), an identical nanoresistor is fabricated for electrothermal actuation of nanomechanical oscillations. $^{\text {? }}$

Our overall approach is as follows. We drive the resonator in several of its eigen-modes shown in the upper inset of Figure la using the electrothermal actuator. The oscillation amplitude of the resonator is carefully calibrated as a function of the drive 
voltage applied to the electrothemal actuator in a heterodyne optical interferometer with a displacement noise floor of $\sim 20$ $\mathrm{fm} / \mathrm{Hz}^{1 / 2}$ at a sample power of $100 \mu \mathrm{W}$. In separate electrical measurements, the piezoresistance is measured during calibrated eigen-mode oscillations. From the oscillation amplitude, the longitudinal strain is calculated numerically and the gauge factor is extracted as a function of (eigen-mode) frequency. We have measured three devices from the same batch with identical strain gauges and embedding circuits (Figures $1 \mathrm{~b}, \mathrm{c}$ ), a $60 \mu \mathrm{m}$-long device, a $50 \mu \mathrm{m}$-long device, and a $30 \mu \mathrm{m}$-long device, with all the relevant parameters listed in Table 2. Further experimental details are provided in the SI. ${ }^{21}$

Table 2. Experimentally-Obtained Mechanical Properties of Measured Devices

\begin{tabular}{crrrr}
$l \times w \times t\left(\mu \mathrm{m}^{3}\right)$ & $n$ & $f_{n}(\mathrm{MHz})$ & $k_{n}(\mathrm{~N} / \mathrm{m})$ & $\chi_{n}\left(\times 10^{-6} / \mathrm{nm}\right)$ \\
$60 \times 0.90 \times 0.1$ & 1 & 4.3 & 6.3 & 1.3 \\
& 2 & 8.9 & 23.2 & 2.7 \\
& 3 & 12.9 & 55.8 & 3.9 \\
$50 \times 0.90 \times 0.1$ & 4 & 17.3 & 93.8 & 5.2 \\
& 1 & 5.2 & 7.4 & 1.6 \\
& 2 & 10.4 & 29.3 & 3.1 \\
$30 \times 0.90 \times 0.1$ & 1 & 15.6 & 69.0 & 4.5 \\
& 4 & 20.8 & 125.0 & 6.1 \\
& 2 & 8.8 & 11.8 & 2.7 \\
& 3 & 26.6 & 98 & 5.3 \\
& 4 & 35.6 & 192 & 10.6 \\
\hline
\end{tabular}

We illustrate the optical calibration of the strain for the fundamental mode of the $50 \mu \mathrm{m}$ resonator. The resonance curves for the mode are shown in Figure 2a. Here, the electrothermal actuator excites the nanomechancial resonance with a harmonic force at different rms amplitudes, with the frequency of the drive force swept around the fundamental mode resonance frequency $f_{1}$ (frequency of the electrical drive swept around $\left.f_{1} / 2\right)$. The $\mathrm{rms}$ oscillation amplitude $\zeta_{1}$ is measured optically at the antinode (i.e., the center). From this measurement, we obtain the mode resonance frequency and quality factor as $f_{1} \approx 5.1825 \mathrm{MHz}$ and $Q_{1} \approx 2.9 \times 10^{4}$, respectively. The inset shows the power spectral density (PSD) of the thermal fluctuations of the same mode of a nominally identical beam, with the integral of the PSD providing the spring constant $k_{1} \approx 7.4 \mathrm{~N} / \mathrm{m}$ from the equipartition of energy. ${ }^{22}$ The mechanical parameters in Table 2 are obtained from similar measurements on other modes, with all the data presented in the SI. ${ }^{21}$ Since the measurements are performed in a vacuum chamber, the quality factors are dependent on the residual pressure in the chamber and are typically high $(5 \times$ $\left.10^{2} \lesssim Q \lesssim 2 \times 10^{4}\right)$. The effect of the $Q$ factor on the measurements is properly removed as discussed below.

Figure $2 \mathrm{~b}$ shows the rms resonance amplitude $\zeta_{1}\left(f_{1}\right)$ as a function of the drive voltage $V_{d}$ for the fundamental mode of the $50 \mu \mathrm{m}$-long resonator. These data are essentially the peak values of the resonance curves, such as those shown in Figure $2 \mathrm{a}$. The solid line in Figure $2 \mathrm{~b}$ is a fit of the form $\zeta_{1}=A_{1} V_{d}^{2}$. The parabolic dependence on voltage arises from the physics of the electrothermal actuator. ${ }^{7}$ The upper inset of Figure $2 \mathrm{~b}$ shows the average longitudinal strain $\bar{\varepsilon}_{x x}$ on the nanoresistor as a function of the resonance amplitude $\zeta_{1}\left(f_{1}\right)$. To find the strain in the nanoresistor due to the bending of the silicon nitride structure, we have resorted to the finite element method
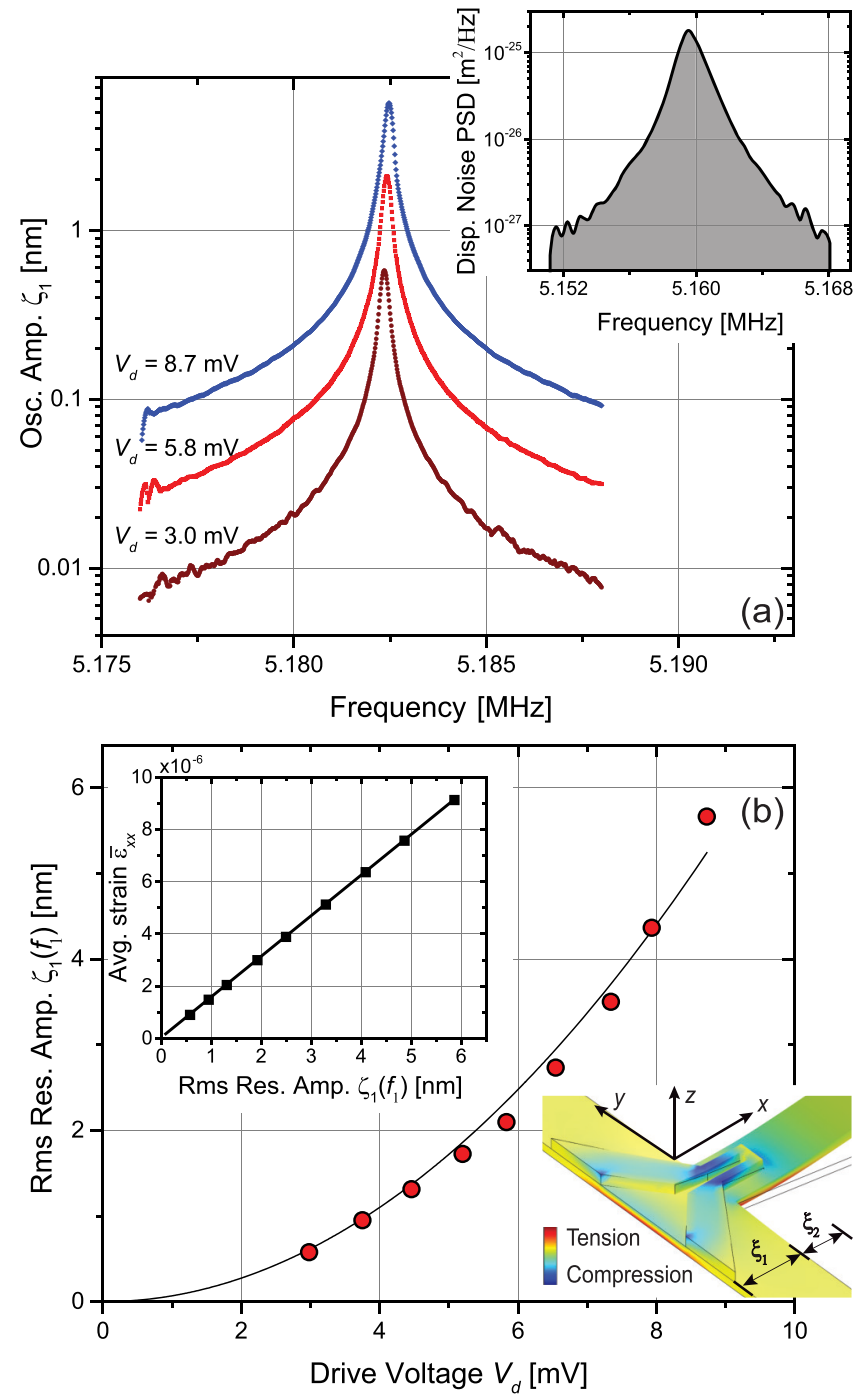

Figure 2. (a) Rms oscillation amplitude of a $50 \mu \mathrm{m}$-long beam at different drives around its fundamental mode resonance frequency measured at its center $(x=l / 2)$. (Inset) power spectral density (PSD) of the Brownian fluctuations of the same mode. (b) Rms resonance amplitude $\zeta_{1}\left(f_{1}\right)$ of the fundamental mode plotted as a function of the drive voltage $V_{d}$. The continuous line is a fit to $\zeta_{1}=A_{1} V_{d}^{2}$. The lower inset is from a finite element model (FEM) showing the relevant strain field $\varepsilon_{x x}(\mathbf{r})$. The upper inset is the average strain $\bar{\varepsilon}_{x x}$ as a function of resonance amplitude $\zeta_{1}\left(f_{1}\right)$ determined from FEM such as the one shown in the lower inset. The strain is linear with amplitude, $\bar{\varepsilon}_{x x}=\chi_{1} \zeta_{1}\left(f_{1}\right)$, with a slope of $1.6 \times 10^{-6} \mathrm{~nm}^{-1}$.

(FEM). Briefly, we solve for the eigen-frequencies of the resonator (including the undercut regions) using boundary mode analysis. Since the resonator is under tension, we apply a tensile load to the silicon nitride layer to match the simulated and experimental eigen-frequencies. We then impose an $\mathrm{rms}$ displacement amplitude for the beam at its antinode and calculate the corresponding strain field. Lower inset of Figure $2 \mathrm{~b}$ shows the rms longitudinal strain field $\varepsilon_{x x}(\mathbf{r})$ as a function of position $\mathbf{r}$ over the suspended base region for an (rms) resonance amplitude of $\zeta_{1}\left(f_{1}\right) \approx 7 \mathrm{~nm}$ (at the center) for the $50 \mu \mathrm{m}$ beam in its fundamental mode. To calculate the average value of $\varepsilon_{x x}(\mathbf{r})$, we first average over the cross-sectional area parallel to the $y z$ plane, $S_{y z}(x)$, of the nanoresistor, finding $\varepsilon_{x x}(x)=\frac{1}{S_{y z}(x)} \iint_{S_{y z}(x)} \varepsilon_{x x}(\mathbf{r}) \mathrm{d} y \mathrm{~d} z$. We ignore the contribution 
(a)

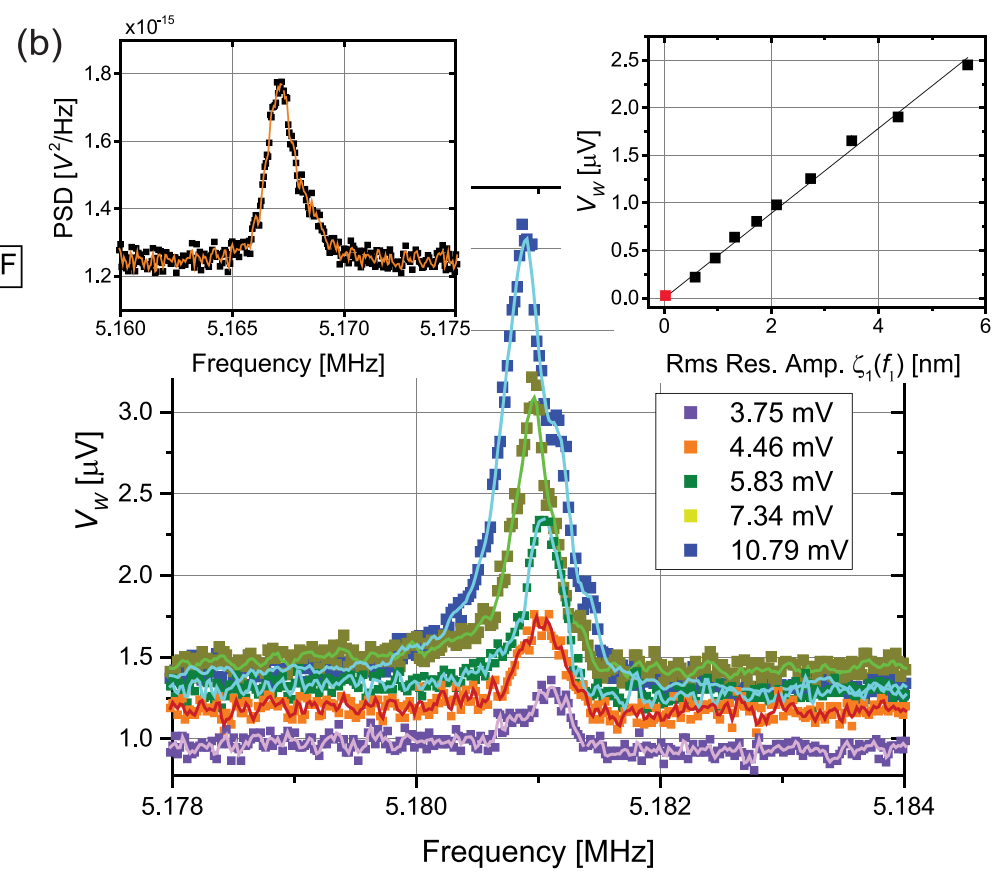

Figure 3. (a) Simplified schematic diagram of the mix-down measurement of piezoresistance. $180^{\circ}$ PS: $180^{\circ}$ power splitter; LPF: low pass filter; FD: frequency doubler. (b) Piezoresistance signal $V_{W}$ at different drives as a function of frequency around the fundamental resonance of the $50 \mu \mathrm{m}-$ long resonator. The bias voltage, $V_{b}=60 \mathrm{mV}$, is kept constant for all curves. Left inset shows the PSD of the Brownian fluctuations of the mode coupling to the piezoresistance signal. Right inset is the measured $V_{W}$ as a function of resonance amplitude of the resonator; all quantities are rms. The red data point is the signal from the Brownian motion. The line is a linear fit through the origin. The error bars in the right inset are smaller than the symbols.

from the small nanoresistor region that is parallel to the $y$ axis. Next, we average $\varepsilon_{x x}(x)$ along the length of the nanoresistor (i.e., $x$ axis). With the origin at the position where the beam structure starts, $\bar{\varepsilon}_{x x}=\frac{1}{\xi_{1}+\xi_{2}} \int_{-\xi_{1}}^{\xi_{2}} \varepsilon_{x x}(x) \mathrm{d} x$. The linear dimensions $\xi_{1}$ and $\xi_{2}$ are shown in Figure $1 \mathrm{~b}$ and the lower inset of Figure $2 \mathrm{~b}$. As a result, we find that, for all modes, $\bar{\varepsilon}_{x x}$ depends linearly on the resonance amplitude of the resonator as $\bar{\varepsilon}_{x x}=$ $\chi_{n} \zeta_{n}\left(f_{n}\right)$ where $\chi_{n}$ is a constant. The results for the fundamental mode of the $50 \mu \mathrm{m}$ beam are shown in the upper inset of Figure $2 \mathrm{~b}$, and all the values of $\chi_{n}$ are listed in Table 2.

Now we turn to the measurement of the piezoresistance signal during driven fundamental eigen-mode oscillations. To reduce parasitic effects, we employ a mix-down measurement ${ }^{23}$ in the balanced circuit ${ }^{24}$ shown in Figure 3a. Briefly, the resonator is driven at its resonance at $f_{n}$ by applying a voltage at $\frac{f_{n}}{2}$ to the electrothermal actuator, ${ }^{7}$ which generates temperature oscillations and hence a thermoleastic force at $f_{n}$. The mechanical strain in the strain gauge causes a timevarying piezoresistance $\sqrt{2} \Delta R \cos \left(2 \pi f_{n} t\right)$. The mix-down and background reduction are accomplished by applying two outof-phase bias voltages of $\pm \sqrt{2} V_{b} \cos \left(2 \pi f_{n} t+2 \pi \Delta f t\right)$ to the two arms of the bridge (ports $A$ and $B$ in Figure $3 a$ and Figure 1c). Assuming negligible imbalance in the bridge $\left(R_{1}=R_{2}\right)$ and $\Delta R \ll R_{1}$, we find the down-converted signal at the input of the measurement electronics (at point $W$ ) as $\sqrt{2} V_{W} \cos (2 \pi \Delta f t)$ where

$$
V_{W}=\frac{V_{b} R_{t} \Delta R}{\sqrt{2}\left(R_{1}+R_{c}+50 \Omega\right)^{2}}
$$

Here, $R_{t}=\left(\frac{2}{R_{1}+R_{c}+50 \Omega}+\frac{1}{R_{z}+R_{c}+50 \Omega}\right)^{-1}$ with $R_{1}=R_{y}+R_{u}$ $+R_{x}$. In our experiments, this signal in eq 1 is detected using a lock-in amplifier referenced to $\Delta f=1.5 \mathrm{MHz} ; V_{b}$ is kept constant. The (rms) value of the piezoresistance $\Delta R$ is then found from the measured $V_{W}$. From separate reflection measurements, we conclude that there is very little attenuation in the bias current $V_{b} /\left(R_{1}+R_{c}+50 \Omega\right)$ and hence the detected signal. The analysis of the detection circuit and complementary measurements (e.g., reflection) are available in the SI. ${ }^{21}$

Figure $3 \mathrm{~b}$ shows the measured rms voltages $V_{W}$ on the strain gauge of the $50-\mu \mathrm{m}$-long beam at different drives as the drive frequency is swept through the fundamental resonance. Compared with the optically detected resonance curves of Figure $2 \mathrm{a}$, one notices that $f_{1}$ and $Q_{1}$ are slightly different. The left inset shows an electrical measurement of the power spectral density (PSD) of the thermal fluctuations of the resonator on the strain gauge. ${ }^{21}$ The right inset shows the rms voltage due to piezoresistance as a function of the nanomechanical resonance amplitude. This voltage is determined by subtracting the baseline value from the peak value at $f_{1}$ in Figure $3 \mathrm{~b}$. The resonance amplitude ( $x$ axis) is determined from the optical calibration in Figure 2 above after accounting for the different $Q$ values in optical and electrical measurements, for example, due to changes in the chamber pressure or resonator surface conditions. Since the amplitude $\zeta_{1}\left(f_{1}\right)$ is given by $\zeta_{1}\left(f_{1}\right)=\frac{F_{1} Q_{1}}{k_{1}}$ with $k_{1}$ a constant and $F_{1}$ only dependent on the applied external voltage, we scale $\zeta_{1}\left(f_{1}\right)$ by the ratio of the $Q$ factors under identical drive voltages. We thus obtain the data in the upper right inset of Figure $3 \mathrm{~b}$. We add the thermal noise result (the red data point in the right inset of Figure $3 \mathrm{~b}$ ) to the $V_{W}$ versus amplitude plot with the 


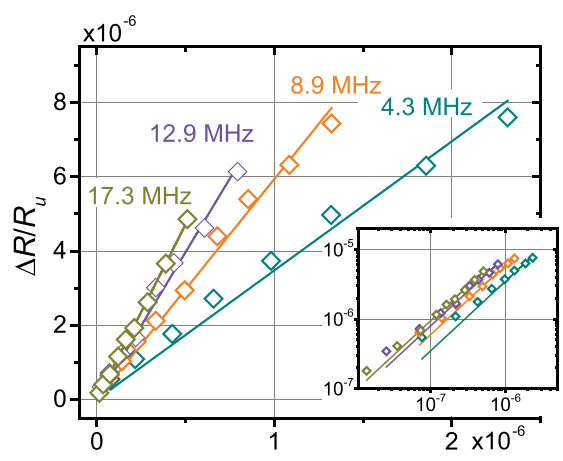

(a)

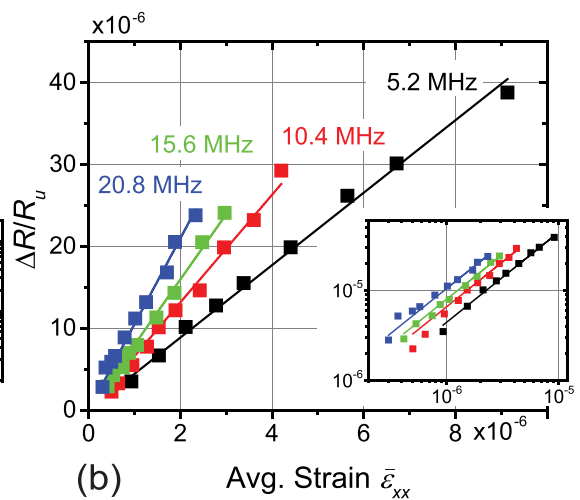

(b)

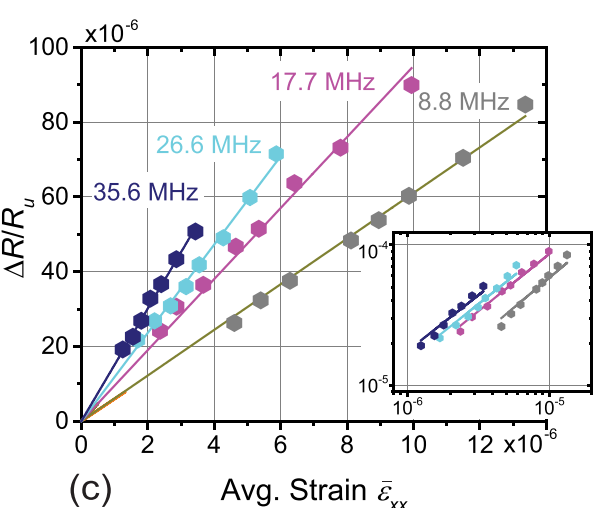

(c)

Figure 4. $\frac{\Delta R}{R}$ as a function of strain $\bar{\varepsilon}_{x x}$ for all the modes of the (a) $60 \mu \mathrm{m}$, (b) $50 \mu \mathrm{m}$, and (c) $30 \mu \mathrm{m}$ resonators. The insets show the same data in double-logarithmic plots. The slopes of the linear fits provide $\gamma$.

understanding that both the voltage and the amplitude are rms quantities obtained from integrals of the PSD. With all the resistances and voltages in the circuit known, it is straightforward to compute the $\Delta R$ values.

Finally, we extract the gauge factor by combining all the measurements. For each mode, we convert the applied drive voltage into resonance amplitude using the optical calibration (Figure 2b main) and the amplitude into strain using the numerical simulations (Figure $2 \mathrm{~b}$ inset). Figures $4 \mathrm{a}, \mathrm{b}$, and $\mathrm{c}$ show $\frac{\Delta R}{R_{u}}$ for the strain gauge as a function of strain for the first four modes of the $60 \mu \mathrm{m}$-long, $50 \mu \mathrm{m}$-long, and $30 \mu \mathrm{m}$-long resonators, respectively. The insets show double-logarithmic plots of the same data. For all modes of the three resonators, $\frac{\Delta R}{R_{u}}$ increases linearly with $\bar{\varepsilon}_{x x}$ with the slope being the gauge factor $\gamma: \frac{\Delta R}{R_{u}}=\gamma \bar{\varepsilon}_{x x}$. We display $\gamma$ for the $60 \mu \mathrm{m}, 50 \mu \mathrm{m}$, and 30 $\mu \mathrm{m}$ resonators as a function of (mode) frequency in Figure 5.

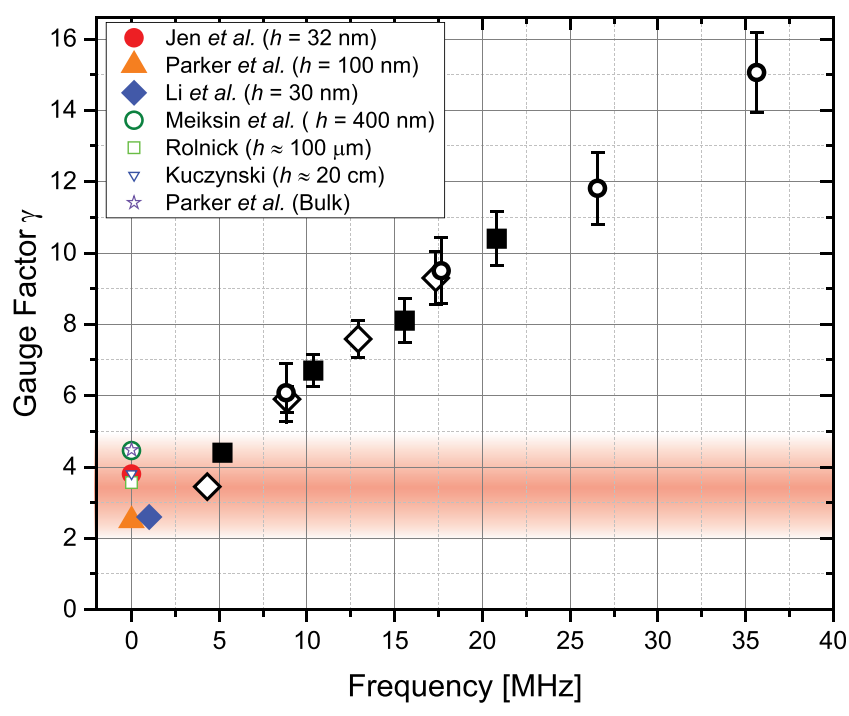

Figure 5. Extracted $\gamma$ as a function of (mode) frequency. The error bars are the rms errors in $\gamma .{ }^{21}$ The error bars at 4.3 and $5.2 \mathrm{MHz}$ are smaller than the symbols. For comparison, we also display previously published $\gamma$ data obtained in quasi-static measurements. The filled data points are for gold films with thicknesses and resistivities comparable to those of our films; ${ }^{10,11,17}$ the open data points are obtained in thick gold films or bulk gold..$^{10,16,25,26}$
The gauge factor increases monotonically from 3.6 to 15 in the frequency range $4.3-36 \mathrm{MHz}$. The error bars in Figure 5 represent rms errors based on standard error analysis discussed in the SI. ${ }^{21}$ Also in Figure 5, we show previously published quasi-static $\gamma$ values for gold films of different thicknesses and for gold wires. The three filled data points around zero frequency show gold films ${ }^{10,11,17}$ with thicknesses and resistivitities (sheet resistances) comparable to those of our films. We also include four data points on very thick films and bulk gold (wires), shown by the open symbols. ${ }^{10,16,25,26}$

Our lowest frequency $\gamma$ value is within the expected range and close to those reported in the literature; this gives us confidence that our resonance-based measurements are accurate. The frequency dependent increase of our $\gamma$, however, is unexpected and cannot be traced to trivial sources, for example, heating or attenuation, which would cause an effect in the opposite direction (i.e., a decrease in $\gamma$ with frequency). We therefore look for possible fundamental mechanisms. The resistivity of our gold films, $\rho=2.81 \times 10^{-8} \Omega \cdot \mathrm{m}$, is close to that of very thick gold films $\left(\rho_{B}=2.44 \times 10^{-8} \Omega \cdot \mathrm{m}\right)^{11,27}$ and bulk gold $\left(\rho_{B}=2.44 \times 10^{-8} \Omega \cdot \mathrm{m}\right){ }^{28-30}$ The slightly larger resistivity of our films compared to $\rho_{B}$ is possibly due to increased surface scattering. Regardless, the electron relaxation time $\tau$ in our gold film at room temperature should be close to the bulk value of $\tau \approx 30 \times 10^{-15} \mathrm{~s}^{31}$ It seems unlikely that an electronic process is the source of the observed effect at the frequencies $f_{n}$ of our experiments, given that $f_{n} \tau \approx 0$. We therefore speculate that mechanical effects give rise to the observed increase. In particular, it is possible that the resonant mechanical motion of the beam couples to a mechanical mode of the nanoresistor or the grains within the nanoresistor. The average grain size in these films is $40 \mathrm{~nm}$, and gold nanorods and nanoparticles of similar dimensions have been shown to have acoustic resonances around $10-100 \mathrm{GHz} .^{32-34}$ Since the gold grains here are in a solid matrix and coupled to other grains mechanically, there could possibly exist lower-frequency mechanical modes within the thin film. Hence, the mechanical energy of the beam may be coupling to these modes and actuating oscillatory strains within the film larger than the strains predicted by FEM. These strains, in turn, may be increasing the grain to grain resistances, giving rise to the observed frequency dependence. This is somewhat similar to the tunneling effects that have been discussed in the piezoresistivity of ultrathin films in which grain to grain transport dominate the piezoresistance. ${ }^{10}$ 
In summary, we have described a method to measure the piezoresistive effect as a function of frequency. More experimental and theoretical studies are needed to pinpoint the source of the observations here. In particular, increasing the frequency range may provide valuable insights. Also, repeating the experiments on strain gauges with different linear dimensions and thicknesses and made up of different metals may help answer some of the questions. Regardless, the effect can be harnessed to develop efficient high-frequency NEMS devices.

\section{ASSOCIATED CONTENT}

\section{(s) Supporting Information}

The Supporting Information is available free of charge at https://pubs.acs.org/doi/10.1021/acs.nanolett.1c01733.

Description of measurement setup and device fabrication process; description of optical measurements, procedure for calibration of strains and spring constants, optical data for all modes of all devices; details of electrical measurements; resistivity and RF measurements on gold film electrodes; analysis of electrical detection circuit; electrical data for all modes of all devices; error analysis (PDF)

\section{AUTHOR INFORMATION}

\section{Corresponding Author}

Kamil L. Ekinci - Department of Mechanical Engineering, Division of Materials Science and Engineering, and the Photonics Center, Boston University, Boston, Massachusetts 02215, United States; 이이.org/0000-0002-5019-5489; Email: ekinci@bu.edu

\section{Authors}

Chaoyang Ti - Department of Mechanical Engineering, Division of Materials Science and Engineering, and the Photonics Center, Boston University, Boston, Massachusetts 02215, United States

Atakan B. Ari - Department of Mechanical Engineering, Division of Materials Science and Engineering, and the Photonics Center, Boston University, Boston, Massachusetts 02215, United States; 이이.orid.org/0000-0002-9587-4338

M. Çagatay Karakan - Department of Mechanical Engineering, Division of Materials Science and Engineering, and the Photonics Center, Boston University, Boston, Massachusetts 02215, United States

Cenk Yanik - SUNUM, Nanotechnology Research and Application Center, Sabanci University, Istanbul 34956, Turkey

Ismet I. Kaya - Faculty of Engineering and Natural Sciences, Sabanci University, Istanbul 34956, Turkey; 이이.org/ 0000-0002-7052-5764

M. Selim Hanay - Department of Mechanical Engineering, Bilkent University, Ankara 06800, Turkey; National Nanotechnology Research Center (UNAM), Bilkent University, Ankara 06800, Turkey

Oleksiy Svitelskiy - Department of Physics, Gordon College, Wenham, Massachusetts 01984, United States

Miguel González - Aramco Services Company, Aramco Research Center-Houston, Houston, Texas 77084, United States

Huseyin Seren - Aramco Services Company, Aramco Research Center-Houston, Houston, Texas 77084, United States
Complete contact information is available at:

https://pubs.acs.org/10.1021/acs.nanolett.1c01733

\section{Notes}

The authors declare no competing financial interest.

\section{ACKNOWLEDGMENTS}

We acknowledge support from Aramco Services Company (A0208-2019) and the US NSF (CBET 1604075, CMMI 1934271, CMMI 2001403, DMR 1709282, and CMMI $1661700)$.

\section{REFERENCES}

(1) Fiorillo, A.; Critello, C.; Pullano, S. Theory, technology and applications of piezoresistive sensors: A review. Sens. Actuators, A 2018, 281, 156-175.

(2) Maluf, N.; Williams, K. Introduction to Microelectromechanical Systems Engineering; Artech House, 2004.

(3) Villanueva, G.; Plaza, J.; Montserrat, J.; Perez-Murano, F.; Bausells, J. Crystalline silicon cantilevers for piezoresistive detection of biomolecular forces. Microelectron. Eng. 2008, 85, 1120-1123.

(4) Chui, B.; Kenny, T.; Mamin, H.; Terris, B.; Rugar, D. Independent detection of vertical and lateral forces with a sidewallimplanted dual-axis piezoresistive cantilever. Appl. Phys. Lett. 1998, 72, $1388-1390$.

(5) Mile, E.; Jourdan, G.; Bargatin, I.; Labarthe, S.; Marcoux, C.; Andreucci, P.; Hentz, S.; Kharrat, C.; Colinet, E.; Duraffourg, L. Inplane nanoelectromechanical resonators based on silicon nanowire piezoresistive detection. Nanotechnology 2010, 21, 165504.

(6) Li, M.; Tang, H. X.; Roukes, M. L. Ultra-sensitive NEMS-based cantilevers for sensing, scanned probe and very high-frequency applications. Nat. Nanotechnol. 2007, 2, 114.

(7) Bargatin, I.; Kozinsky, I.; Roukes, M. Efficient electrothermal actuation of multiple modes of high-frequency nanoelectromechanical resonators. Appl. Phys. Lett. 2007, 90, 093116.

(8) Kouh, T.; Hanay, M. S.; Ekinci, K. L. Nanomechanical motion transducers for miniaturized mechanical systems. Micromachines 2017, $8,108$.

(9) Tang, H.; Li, M.; Roukes, M. L. Metallic thin film piezoresistive transduction in micromechanical and nanomechanical devices and its application in self-sensing SPM probes. US Patent US7617736, 2009.

(10) Parker, R.; Krinsky, A. Electrical Resistance-Strain Characteristics of Thin Evaporated Metal Films. J. Appl. Phys. 1963, 34, 27002708.

(11) Jen, S.; Yu, C.; Liu, C.; Lee, G. Piezoresistance and electrical resistivity of $\mathrm{Pd}, \mathrm{Au}$, and $\mathrm{Cu}$ films. Thin Solid Films 2003, 434, 316322.

(12) Neugebauer, C.; Webb, M. Electrical conduction mechanism in ultrathin, evaporated metal films. J. Appl. Phys. 1962, 33, 74-82.

(13) Verma, B.; Juretschke, H. Strain dependence of the resistivity of silver films. J. Appl. Phys. 1970, 41, 4732-4735.

(14) Verma, B.; Jain, G. Size effect in longitudinal and transverse strain coefficient of resistance in silver films. Thin Solid Films 1972, $11,27-32$.

(15) Tellier, C.; Tosser, A. Grain Size Dependence of the Gauge Factor of Thin Metallic Films. Electrocomponent Sci. Technol. 1977, 4, 9-17.

(16) Kuczynski, G. Effect of elastic strain on the electrical resistance of metals. Phys. Rev. 1954, 94, 61.

(17) Li, C.; Hesketh, P.; Maclay, G. Thin gold film strain gauges. J. Vac. Sci. Technol., A 1994, 12, 813-819.

(18) He, R.; Feng, X.; Roukes, M.; Yang, P. Self-transducing silicon nanowire electromechanical systems at room temperature. Nano Lett. 2008, 8, 1756-1761.

(19) Phan, H.-P.; Kozeki, T.; Dinh, T.; Fujii, T.; Qamar, A.; Zhu, Y.; Namazu, T.; Nguyen, N.-T.; Dao, D. V.; et al. Piezoresistive effect of p-type silicon nanowires fabricated by a top-down process using FIB implantation and wet etching. RSC Adv. 2015, 5, 82121-82126. 
(20) Neuzil, P.; Wong, C. C.; Reboud, J. Electrically controlled giant piezoresistance in silicon nanowires. Nano Lett. 2010, 10, 1248-1252.

(21) See Supporting Information for additional details and data.

(22) Ari, A. B.; Hanay, M. S.; Paul, M. R.; Ekinci, K. L. Nanomechanical measurement of the brownian force noise in a viscous liquid. Nano Lett. 2021, 21, 375-381.

(23) Bargatin, I.; Myers, E.; Arlett, J.; Gudlewski, B.; Roukes, M. Sensitive detection of nanomechanical motion using piezoresistive signal downmixing. Appl. Phys. Lett. 2005, 86, 133109.

(24) Ti, C.; Ari, A.; Orhan, E.; Gonzalez, M.; Yanik, C.; Kaya, I. I.; Hanay, M. S.; Ekinci, K. L. Optimization of Piezoresistive Motion Detection for Ambient NEMS Applications; IEEE Sensors, 2020; pp 14.

(25) Meiksin, Z.; Hudzinski, R. A theoretical study of the effect of elastic strain on the electrical resistance of thin metal films. J. Appl. Phys. 1967, 38, 4490-4494.

(26) Rolnick, H. Tension coefficient of resistance of metals. Phys. Rev. 1930, 36, 506.

(27) Chopra, K.; Bobb, L.; Francombe, M. Electrical resistivity of thin single-crystal gold films. J. Appl. Phys. 1963, 34, 1699-1702.

(28) Graz, I. M.; Cotton, D. P.; Lacour, S. P. Extended cyclic uniaxial loading of stretchable gold thin-films on elastomeric substrates. Appl. Phys. Lett. 2009, 94, 071902.

(29) Goodman, P. Current and future uses of gold in electronics. Gold bulletin 2002, 35, 21-26.

(30) Christie, I. R.; Cameron, B. P. Gold electrodeposition within the electronics industry. Gold Bulletin 1994, 27, 12-20.

(31) Gall, D. Electron mean free path in elemental metals. J. Appl. Phys. 2016, 119, 085101.

(32) Zijlstra, P.; Tchebotareva, A. L.; Chon, J. W.; Gu, M.; Orrit, M. Acoustic oscillations and elastic moduli of single gold nanorods. Nano Lett. 2008, 8, 3493-3497.

(33) Pelton, M.; Sader, J. E.; Burgin, J.; Liu, M.; Guyot-Sionnest, P.; Gosztola, D. Damping of acoustic vibrations in gold nanoparticles. Nat. Nanotechnol. 2009, 4, 492-495.

(34) Ruijgrok, P. V.; Zijlstra, P.; Tchebotareva, A. L.; Orrit, M. Damping of acoustic vibrations of single gold nanoparticles optically trapped in water. Nano Lett. 2012, 12, 1063-1069. 